\title{
sciendo
}

\section{A case report of simultaneous medullary and papillary carcinoma of thyroid}

\author{
Reza Ziaolhagh ${ }^{1}$, Ali SadrizadeH ${ }^{2}$, Babak Peyro Shabany ${ }^{3}$, Asma Ahrari Roudi ${ }^{4}$ \\ ${ }^{1}$ Razavi Hospital, Mashhad, Iran; ${ }^{2}$ Lung Diseases Research Center, Mashhad University of Medical Sciences, Mashhad, \\ Iran; ${ }^{3}$ Department of Internal Medicine, Sabzevar University of Medical Sciences, Sabzevar, Iran; ${ }^{4}$ Cellular and \\ Molecular Research Center, Sabzevar University of Medical Sciences, Sabzevar, Iran \\ E-mail: AhrariA951@mums.ac
}

Objective. Medullary (MTC) and papillary (PTC) thyroid carcinoma are two different types of thyroid carcinoma with significant differences in origin. Their co-occurrence in a patient is a rare phenomenon. We report a patient with simultaneous presentation of both MTC and PTC.

Case presentation. A 62-year-old euthyroid woman with a cervical mass was evaluated, underwent total thyroidectomy, and neck dissection. The examination revealed a MTC large nodule as well as a small nodule of the tall cell variant of PTC, along with the concomitant cervical lymph node metastases. Subsequently, the genetic analysis showed BRAF mutations. Adjuvant treatments including radioiodine and thyroid hormone replacement therapies were performed for the patient.

Conclusions. The cooccurrence of MTC and PTC in the same patient is a rare phenomenon. The clinical manifestations and biological behavior of these cancers are completely different. Since the therapeutic strategy and prognosis are very different in these patients, accurate diagnosis of this coexistence is very important.

Key words: medullary thyroid cancer, papillary thyroid cancer, BRAF mutations

Thyroid cancer is the most common neuroendocrine tumor (Davies and Welch 2006; Pellegriti et al. 2013). The increased incidence of thyroid cancer is substantially attributed to the diagnosis of small and subclinical nodules, which are now found through the exact ultrasonographic evaluation (Gurkan et al. 2014; Jain et al. 2014; Dikbas et al. 2019). Medullary (MTC) and papillary (PTC) thyroid carcinoma are two main subgroups of thyroid cancer. PTC, which its different variants constitute approximately $80 \%$ of all thyroid carcinomas (Moslehi et al. 2016), is mainly originated in thyroid follicular cells (the major cell type in the thyroid gland), which production of thyroid hormones is their main role (Dikbas et al. 2019).
MTC, which forms five to eight percent of thyroid cancers (Ball 2007), has a lot of cytoarchitectural variations and drives from the parafollicular $\mathrm{C}$ cells of the thyroid gland, which produce and secrete calcitonin and other peptide hormones (Ball 2007; Guilmette and Nose 2018). MTC, organized in nested or infiltrative patterns, may appear in spindle, oxyphil, small, and even anaplastic cell types. In addition, the amyloid deposition has been reported in MTC (Kakudo et al. 2018). The cooccurrence of MTC and PTC is rare. However, in some clinical scenarios, the presence of both tumors in a single or two separated lesions with different pathological mechanisms has been reported (Lamberg et al. 1981; Darwish et al. 1995; Sadat Alavi and Azarpira 2011). In this case

Corresponding author: Dr. Asma Ahrari Roudi, Vasei Educational Hospital, Sabzevar University of Medical Sciences, TohidShar BLV, Sabzevar, Razavi Khorasan, 9617747431, Iran; phone: +98 51 44014091; fax: +98 51 44653861; e-mail: AhrariA951@mums.ac.ir. 
study, we have presented a patient with simultaneous incidence of both MTC and PTC, accompanied by concurrent metastasis of both of the cervical lymph nodes.

\section{Case presentation}

A 62-year-old Iranian female patient presented with an anterior cervical mass. The patient had no history of head and neck radiation exposure and no family history of any endocrine disease. During the physical examination, a large, fixed, and well-defined nodule was detected. Ultrasound examination of the neck showed a solid and hyperechoic nodule measuring $95 \times 50 \mathrm{~mm}$ in the right lobe of thyroid and also a mixed echoic nodule at the left lobe, measuring $25 \mathrm{~mm}$. Besides, several cervical lymphadenopathies were detected on both sides of the neck. The thyroid hormones and thyroid stimulating hormone (TSH) levels were in the normal range and also there was no pathological findings on sonographic evaluation of abdomen.

Fine needle aspiration from thyroid nodule showed hypercellular smear with multiple loosely cohesive cellular clusters and isolated round cells with mildly pleomorphic round nuclei and eosinophilic cytoplasm in consistent with medullary carcinoma. Preoperative serum level of calcitonin was very high $(>2000 \mathrm{pg} / \mathrm{ml})$ [reference value in females: up to 4.8]. The patient was evaluated for multiple endocrine neoplasia, which had negative results as well as the calcium and parathyroid hormone levels were normal.

The patient underwent a total thyroidectomy with bilateral modified radical neck dissection, revealing a creamy and well circumscribed nodule in the right lobe with extending to the left lobe, measuring
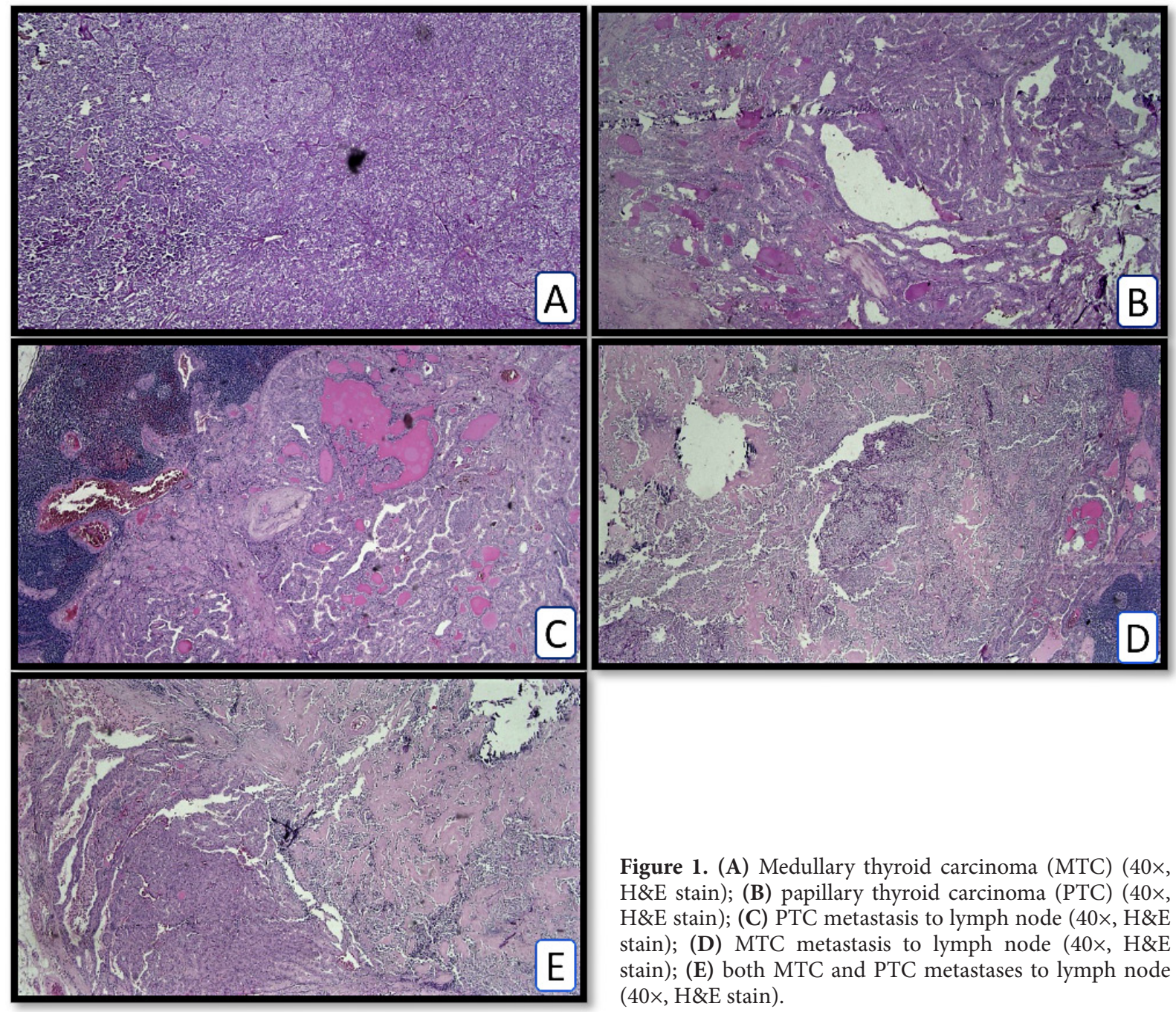

Figure 1. (A) Medullary thyroid carcinoma (MTC) (40x, H\&E stain); (B) papillary thyroid carcinoma (PTC) (40×, H\&E stain); (C) PTC metastasis to lymph node (40×, H\&E stain); (D) MTC metastasis to lymph node $(40 \times, H \& E$ stain); (E) both MTC and PTC metastases to lymph node (40×, H\&E stain). 
$7.7 \times 5.7 \mathrm{~cm}$ and also a creamy nodule measuring $3.3 \times 2.1 \mathrm{~cm}$ on the left one.

Light microscopic assessment showed that the two nodules had completely different morphologies. The larger tumor in the right lobe had a nesting growth pattern composed of round and spindle cells with mildly pleomorphic round to oval nuclei and abundant eosinophilic cytoplasm. The mitotic activity was low and the stroma contained a homogenous and pink ground substance (Figure 1A). Tumor cells were positive in immunohistochemistry for calcitonin, synaptophysin, chromogranin A, carcinoembyronic antigen (CEA), PAX8, thyroid transcription factor 1 (TTF-1) (Figures 2A-F), while the result for thyroglobulin (TG) was negative (Figure $2 \mathrm{G}$ ). Consequently, the MTC was reported.
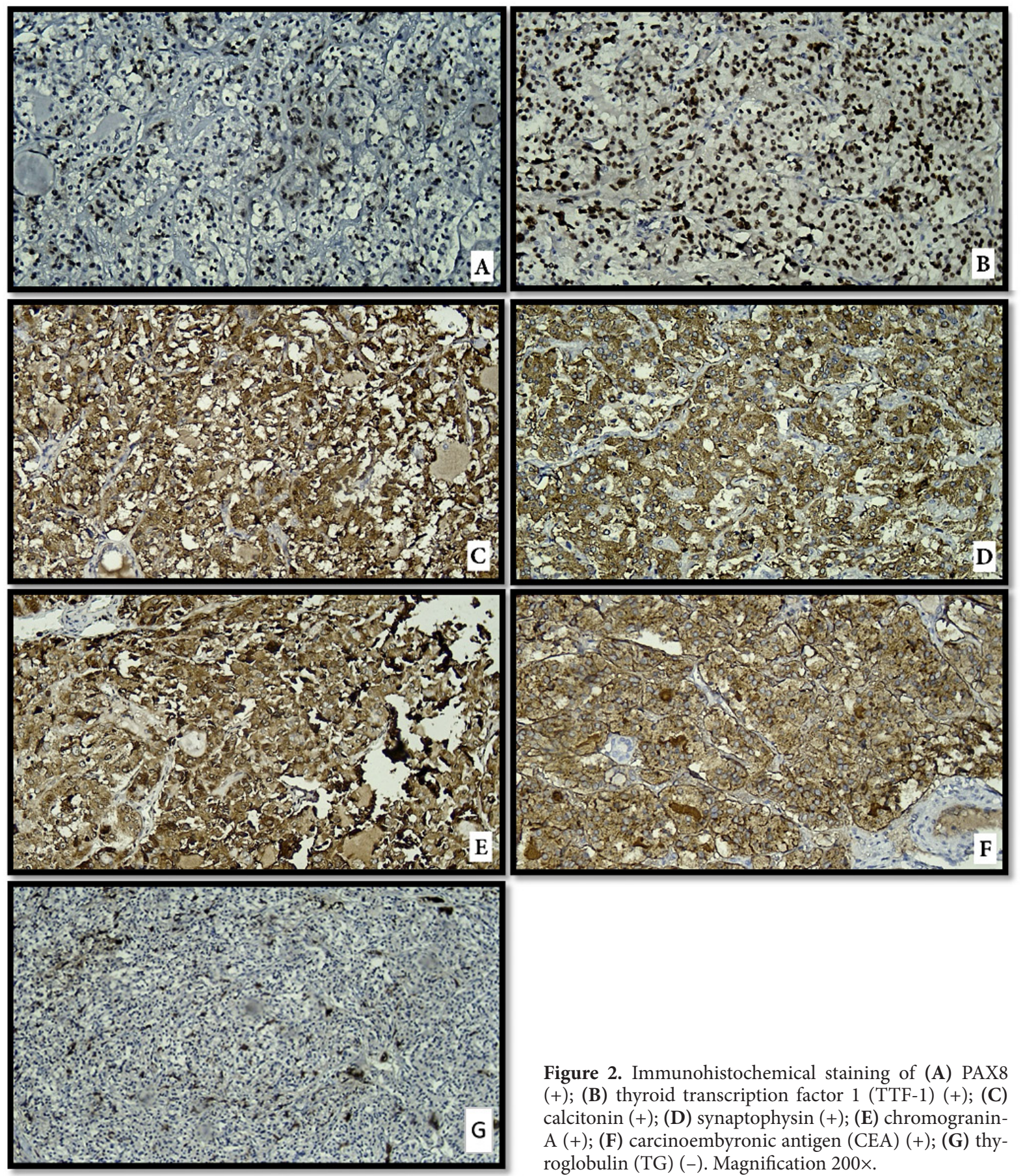

Figure 2. Immunohistochemical staining of (A) PAX8 (+); (B) thyroid transcription factor 1 (TTF-1) (+); (C) calcitonin (+); (D) synaptophysin (+); (E) chromograninA (+); (F) carcinoembyronic antigen (CEA) $(+)$; (G) thyroglobulin (TG) (-). Magnification 200×. 
The smaller tumor in the left lobe was composed of follicular and papillary structures, lined by tall columnar cells with nuclear clearing, grooving and occasional pseudoinclusion (Figure 1B). These cells were positive in immunohistochemistry for TTF-1, PAX8, TG (Figures 3A-C) and negative for calcitonin, synaptophysin, chromogranin A (Figures 3D-F). As a result, a tall cell variant of PTC was reported.

In the microscopic examination of the left lateral cervical lymph nodes, 6 of the 17 only had PTC metastasis (Figure 1C). In the central cervical lymph nodes, 6 of the 11 nodes were found to had MTC metastasis (Figure 1D), 2 nodes had PTC metastases, and in one lymph node both of the MTC and PTC metastases were seen (Figure 1E). In the genetic examination of PTC, a BRAFV600E somatic mutation was seen
(c.1799>A). Accompanied with thyroid hormones, the patient received iodine 131 for the ablation of the remnant of thyroid tissue.

\section{Discussion}

The co-occurrence of MTC and PTC in the same thyroid gland is a rare phenomenon and is divided into two forms. New developments in the molecular assessments of tumors have led to the exact diagnosis of these rare situations in the recent years (Javadinia et al. 2018a,b; Fanipakdel et al. 2019). The first form consists of two distinct tumors that are separated by non-neoplastic thyroid tissue. The other one is a mixed tumor with dual differentiations, which our patient had first form (Rossi et al. 2005). Based on
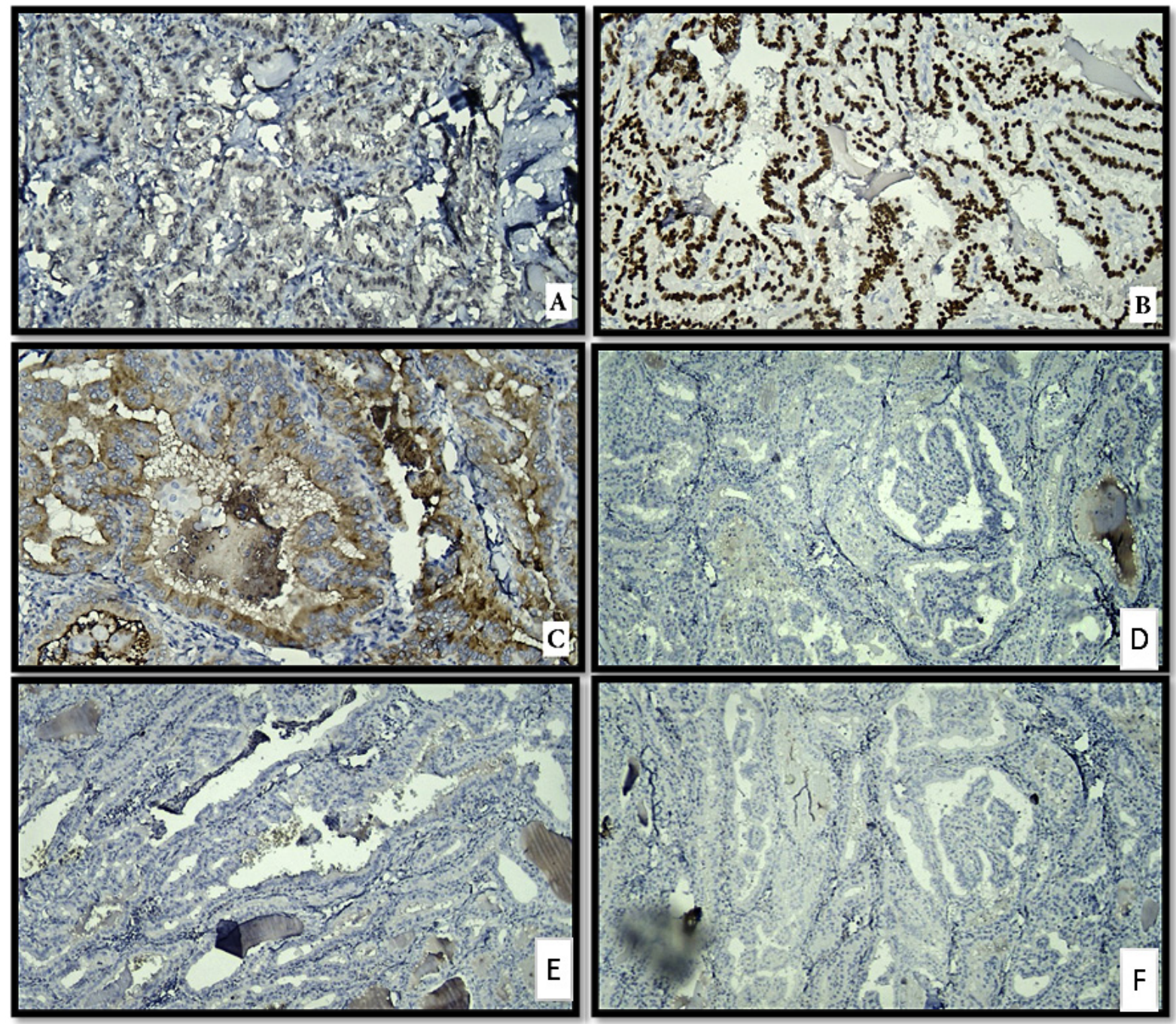

Figure 3. Immunohistochemical staining of (A) PAX8 (+); (B) thyroid transcription factor 1 (TTF-1) (+); (C) thyroglobulin (TG) (+); (D) calcitonin (-); (E) synaptophysin (-); (F) chromogranin-A (-). Magnification 200x. 
histopathological and immunohistochemical findings, larger nodule was positive for calcitonin, synaptophysin, chromogranin A, CEA, PAX8 and TTF-1, confirming the diagnosis of MTC. In other hand, the smaller nodule was positive for PAX8 and TTF-1 which is a characteristic diagnosis of tall cell variant of PTC.

In MTC, PTC, and in the case of co-occurrence of both there can be metastases to the cervical lymph nodes (Sadat Alavi and Azarpira 2011). In our case, a combination of all forms was reported the patient's lymph nodes. The co-occurrence of these two tumors is more common in women $(\mathrm{M} / \mathrm{F}=1 / 2)$, and in the patients in the 6th and 7th decades of their lives (Rossi et al. 2005).

The histogenetic etiology and molecular mechanisms leading to mixed MTC and PTC have not been determined yet. The most commonly accepted hypotheses are the tumor stem cell, collision effect, and hostage theories (Gurkan et al. 2014). The MTC can appear sporadic or familial. The inherited familial type is in three forms: MEN2A syndrome,
MEN2B syndrome, and negative for MEN2. The patients suffering from MTC with a negative family history should be screened for MEN2. However, the evaluation of RET oncogene for MTC in different studies shows different results. Genetic analysis for BRAF POINT MUTATION shows a higher specificity. Results show BRAFV600E (c.1799>A).

\section{Conclusion}

The cooccurrence of MTC and PTC in the same patient is a rare phenomenon. The clinical manifestations and biological behavior of these cancers are completely different. Since the therapeutic strategy and prognosis is very different in these patients, accurate diagnosis of this coexistence is very important. PTC responds to the TSH suppressing and the radioiodine therapies. However, due to the nonresponsivity of MTC to radioactive iodine, radiation treatments, chemotherapy, and surgery without adjuvant therapy is suggestive as the major treatment.

\section{References}

Ball DW. Medullary thyroid cancer: therapeutic targets and molecular markers. Curr Opin Oncol 19, 18-23, 2007.

Darwish A, Satir AA, Hameed T, Malik S, Aqel N. Simultaneous medullary carcinoma, occult papillary carcinoma and lymphocytic thyroiditis. Malays J Pathol 17, 103-107, 1995.

Davies L, Welch HG. Increasing incidence of thyroid cancer in the United States, 1973-2002. JAMA 295, 2164-2167, 2006.

Dikbas O, Duman AA, Guvendi GF. Medullary thyroid carcinoma and papillary thyroid carcinoma in the same patient as a Collision tumour. Case Rep Endocrinol 2019, 4038628, 2019.

Fanipakdel A, Seilanian Toussi M, Rezazadeh F, Mohamadian Roshan N, Javadinia SA. Overexpression of cancertestis antigen melanoma-associated antigen A1 in lung cancer: A novel biomarker for prognosis, and a possible target for immunotherapy. J Cell Physiol 234, 12080-12086, 2019.

Guilmette J, Nose V. Hereditary and familial thyroid tumours. Histopathology 72, 70-81, 2018.

Gurkan E, Gurbuz Y, Tarkun I, Canturk Z, Cetinarslan B. Mixed medullary-papillary carcinoma of the thyroid: report of two cases and review of the literature. Indian J Pathol Microbiol 57, 598-602, 2014.

Jain M, Verma D, Thomas S, Chauhan R. Mixed medullary - papillary carcinoma thyroid: an uncommon variant of thyroid carcinoma. J Lab Physicians 6, 133-135, 2014.

Javadinia SA, Shahidsales S, Fanipakdel A, Joudi-Mashhad M, Mehramiz M, Talebian S, Maftouh M, Mardani R, Hassanian SM, Khazaei M, Ferns GA, Avan A. Therapeutic potential of targeting the Wnt/ $\beta$-catenin pathway in the treatment of pancreatic cancer. J Cell Biochem 2018. Epub ahead of print.

Javadinia SA, Shahidsales S, Fanipakdel A, Mostafapour A, Joudi-Mashhad M, Ferns GA, Avan A. The esophageal cancer and the PI3K/AKT/mTOR signaling regulatory microRNAs: a novel marker for prognosis, and a possible target for immunotherapy. Curr Pharm Des 24, 4646-4651, 2018.

Kakudo K, Bychkov A, Bai Y, Li Y, Liu Z, Jung CK. The new 4th edition World Health Organization classification for thyroid tumors, Asian perspectives. Pathol Int 68, 641-664, 2018.

Lamberg BA, Reissel P, Stenman S, Koivuniemi A, Ekbolm M, Makinen J, Franssila K. Concurrent medullary and papillary thyroid carcinoma in the same thyroid lobe and in siblings. Acta Med Scand 209, 421-424, 1981.

Moslehi M, Shahi Z, Badihian S, Jalalpour P, Sedghian M, Motamedi G, Tavakol G. Differentiated thyroid cancer in Iran - initial observations, histological features, management of the disease, and tumor recurrence: A review of 1689 cases. Indian J Cancer 53, 261-264, 2016. 
Pellegriti G, Frasca F, Regalbuto C, Squatrito S, Vigneri R. Worldwide increasing incidence of thyroid cancer: update on epidemiology and risk factors. J Cancer Epidemiol 2013, 965212, 2013.

Rossi S, Fugazzola L, De Pasquale L, Braidotti P, Cirello V, Beck-Peccoz P, Bosari S, Bastagli A. Medullary and papillary carcinoma of the thyroid gland occurring as a collision tumour: report of three cases with molecular analysis and review of the literature. Endocr Relat Cancer 12, 281-289, 2005.

Sadat Alavi M, Azarpira N. Medullary and papillary carcinoma of the thyroid gland occurring as a collision tumor with lymph node metastasis: A case report. J Med Case Rep 5, 590, 2011. 